\title{
Physicochemical characterization of astaxanthin-loaded PLGA formulation via nanoprecipitation technique
}

\author{
Ku Aizuddin Ku Azman ${ }^{1}$, Foo Chit Seong ${ }^{2}$, Gurmeet Kaur Surindar Singh ${ }^{3}$, Meor Mohd Redzuan Meor Mohd Affandi ${ }^{*}$ \\ ${ }^{1}$ Unit Biologi, Jabatan Sains, MRSM Serting, Bandar Baru Serting, Bandar Seri Jempol, Negeri Sembilan, Malaysia. \\ ${ }^{2} \mathrm{MBH}$ Cosmeceutical Sdn. Bhd, Kapar, Klang, Selangor, Malaysia. \\ ${ }^{3}$ Department of Pharmaceutical Life Sciences, Faculty of Pharmacy, Universiti Teknologi MARA (UiTM), Puncak Alam Campus, Bandar Puncak Alam, \\ Selangor Darul Ehsan, Malaysia. \\ ${ }^{4}$ Department of Pharmaceutics, Faculty of Pharmacy, Universiti Teknologi MARA (UiTM), Puncak Alam Campus, Bandar Puncak Alam, Selangor Darul \\ Ehsan, Malaysia.
}

\section{ARTICLE INFO \\ Received on: 26/11/2020 \\ Accepted on: 26/02/2021 \\ Available online: 05/06/2021}

\section{Key words:}

Astaxanthin, bioavailability, nanoparticles,

physicochemical

characteristics. PLGA.

\begin{abstract}
Astaxanthin is a strong antioxidant with numerous medicinal properties. However, it is highly lipophilic and has low bioavailability which hinders its absorption capabilities. In order to improve the absorption of astaxanthin, the present study evaluates the physicochemical characteristics of astaxanthin-loaded poly(lactic-co-glycolic acid) (PLGA) nanoparticles prepared via the nanoprecipitation technique. An optimum formulation of nanoparticles was then tested for particle size, zeta potential, encapsulation and entrapment efficiency, morphology, thermal behavior, crystallinity, and compound-polymer interaction. The size of the particles and zeta potential of the formed nanoparticle were reported at $142.23 \pm 0.961 \mathrm{~nm}$ and $-27.3 \pm 4.67 \mathrm{mV}$, respectively. Scanning electron microscope (SEM) study through photomicrogram confirmed the nano-range size of the optimum formulation. The formulation shows low nanoparticle yield but recorded high encapsulation efficiency. Density scanning calorimetry and X-ray diffraction results proved that the nanoparticles were in an amorphous state. Fourier transform infrared spectroscopy analysis had shown that the stretching vibrations of ketone and hydroxyl functional groups were restricted, indicating that the astaxanthin was encapsulated inside the PLGA polymer. These findings provide valuable information regarding astaxanthin bioavailability enhancement through the nanoprecipitation technique.
\end{abstract}

\section{INTRODUCTION}

Free radicals are yields of normal cellular activities. They play an important role in the biological function of humans in which they may assist immunity by mediating cell signaling and play an important role in apoptosis. However, free radicals may lead to the impairment of important macromolecules in cells and have been shown to be involved in carcinogenesis and cardiovascular diseases (Sailaja Rao et al., 2011; Tvrdá and Benko, 2020). Various reports have demonstrated the production of free radicals as an initial response to both pathogenic and

\footnotetext{
${ }^{*}$ Corresponding Author

Meor Mohd Redzuan Meor Mohd Affandi, Department of Pharmaceutics, Faculty of Pharmacy, Universiti Teknologi MARA (UiTM), Puncak Alam Campus, Bandar Puncak Alam, Selangor Darul Ehsan, Malaysia.

E-mail:meor@uitm.edu.my
}

nonpathogenic microorganisms in plants (Macarisin et al., 2010). Although known as mediators of intracellular signaling cascades, excessive production of free radicals may lead to a loss of cell function and eventually apoptosis (Alfarouk et al., 2020). Another condition that is usually caused by the excessive production of free radicals is oxidative stress. Oxidative stress is the discrepancy between free radical development and the body's antioxidant defense capacity (Lobo et al., 2010). Although the human body system is capable of generating its own antioxidants to fight against free radicals, the production will plunge as the human body ages.

Astaxanthin is well known for being a strong antioxidant. The antioxidant capacity of astaxanthin is approximately 10 times stronger than zeaxanthin, lutein, $\beta$-carotene, and tunaxanthin and is 100 times greater than $\alpha$-tocopherol (Inoue et al., 2019). The substance belongs to the xanthophylls group and does not possess a vitamin A activity (Meor Mohd Affandi, et al., 2012; Tolba et al., 2020). Astaxanthin is rich in antioxidant activity, is able to esterify, and is more polar than other carotenoids. 
These are some of the substance's distinctive features owing to the presence of hydroxyl $(\mathrm{OH})$ and ketone group $(\mathrm{C}=\mathrm{O})$ on its ionone ring (Meor Mohd Affandi et al., 2011). Numerous reports have illustrated various medicinal potentials of astaxanthin such as chemopreventive effects, anti-inflammatory properties, skin protection from damaging effects of ultraviolet, and the reduction in age-related macular degeneration (Ito et al., 2018; Kavitha et al., 2013; Li et al., 2020; Yasui et al., 2011). Astaxanthin is strongly lipophilic and has poor oral bioavailability, despite being a potent antioxidant, similar to other carotenoids (Yang et al., 2020). There has been a surge of interest among researchers to unravel the best approach to boost astaxanthin absorption in the human body. For diagnostic and therapeutic uses, there are many techniques that can be used in pharmaceutical applications, such as carbon nanotubes, liposomes, micelles, polymer nanoparticles, and metallic nanoparticles. Among them, one promising approach to overcome the low bioavailability of astaxanthin is by incorporating the substance in nanoparticles by using certain polymers (polymeric nanoparticles). Nanoparticles have been reported to enhance drug delivery to the target site, control drug release, and reduce drug-associated adverse effects.

In this study, poly(lactic-co-glycolic acid) (PLGA) was selected due to its biodegradable, biocompatible, and nontoxic characteristics (Fredenberg et al., 2011). Previously, we have successfully formulated astaxanthin-loaded PLGA nanoparticles (average mean size of $0.231 \pm 0.006$ ) through the nanoprecipitation technique (Ku Aizuddin et al., 2014). However, the development of an optimized formulation is not sufficient without the support of a physicochemical properties study. Conducting physicochemical characterization of nanoparticles is detrimental to uncover the reaction of the biological system toward these nanosized particles, particularly the size and surface area of nanoparticles which are two prominent deciding factors for nanoparticle's fate in the body. Absorption, distribution, retention, and removal of nanoparticles are dependent on particle size and surface which are important in their formulation. Other than that, the structural state of nanoparticles is also important to determine their solubility and, by an extent, their bioavailability. Nanoparticle characterization is also valuable in the industry for scaling up (Raval et al., 2019). A previous physicochemical study has used the emulsion solvent evaporation technique but to the best of our knowledge the nanoprecipitation technique is yet to be explored (Hu et al., 2019). Therefore, this study is aimed to evaluate the physicochemical characterization of the newly designed nanoparticles proposed for the oral route of administration. Assessment on entrapment efficiency, nanoparticle recovery, morphology, particle size, zeta potential, thermal properties, x-ray diffraction (XRD), and fourier transform infrared (FTIR) spectroscopy was conducted. The findings of this study will provide important information on the physicochemical characterization of astaxanthin-loaded PLGA formulation via nanoprecipitation technique as an alternative method to improve astaxanthin bioavailability.

\section{EXPERIMENTAL METHOD}

\section{Materials}

Astaxanthin was purchased from Sigma-Aldrich Chemical (St. Louis, MO). PLGA with a molecular weight ranging from 7,000 to $240,000 \mathrm{kDa}$ (lactide:glycolide 50:50) was supplied by Boehringer Ingelheim (Ingelheim, Germany). The surfactant Pluronic F-127 and analytical grade acetone were procured from Sigma-Aldrich Chemical and Merck (Darmstadt, Germany), respectively.

\section{Preparation of nanoparticles}

The nanoprecipitation technique was used to prepare astaxanthin-loaded PLGA as discussed in our previous work $(\mathrm{Ku}$ Aizuddin et al., 2014). The surfactant was dissolved in water as an aqueous phase. Meanwhile, PLGA and astaxanthin were dissolved in acetone as an organic phase. In a dropwise manner $(1 \mathrm{ml}$ second $d^{-1}$, the aqueous phase was added to the organic phase and mixed with a magnetic stirrer at the speed of 2 . Then, the solvent was left evaporate for 90 minutes. For 15 minutes, the freshly prepared nanoparticles were then centrifuged twice at 5,000 rpm. The pellet was washed twice with $5 \mathrm{ml}$ distilled water in which the astaxanthin, polymer, and surfactant were dissolved.

\section{Physicochemical study of astaxanthin-loaded PLGA nanoparticles}

Particle size and zeta potential

The particle size, polydispersity index (PDI), and zeta potential were evaluated by using the Zetasizer 1,600 (Malvern Instruments Ltd, Malvern, UK). Both size and zeta potential determination was carried out in triplicate following the dispersion of nanoparticles in distilled water at $25^{\circ} \mathrm{C}$. The average particle size was expressed in mean diameter and the reported value was represented as $\pm \mathrm{SD}(n=3)$.

\section{Scanning electron microscope (SEM)}

The morphology of astaxanthin biodegradable nanoparticles was analyzed with environmental SEM (FEI Quanta 450 FEG). One drop of the dispersed nanoparticles was placed on the carbon-coated grid. The test was carried out at room temperature.

\section{Nanoparticle yield and entrapment efficiency}

Freeze-dried astaxanthin-loaded PLGA nanoparticles were accurately weighed and the nanoparticle recovery (\%) was calculated using Equation (1). Meanwhile, the entrapment efficiency of astaxanthin-loaded PLGA nanoparticles was also calculated using Equation (2). All calculations and values were made in triplicate.

(1) Nanoparticle recovery $(\%)=($ mass of nanoparticle $) /($ mass of polymer, surfactant and drug used $) \times 100$

(2) Entrapment efficiency (\%) = (mass of drug in nanoparticle)/ (mass of drug used in formulation) $\times 100$

\section{Differential scanning calorimetry (DSC)}

Astaxanthin-loaded PLGA nanoparticles were analyzed with DSC 8,500 (Perkin Elmer Inc., America). Powdered PLGA, pure astaxanthin, and astaxanthin-loaded PLGA nanoparticles were placed in a separate pan and their tested weight was recorded. The samples were then crimpled before the test resumed. The entire DSC tests were carried out in triplicate (scanning rate: $10^{\circ} \mathrm{C}$ second ${ }^{-1}$, $0^{\circ} \mathrm{C}-250^{\circ} \mathrm{C}$ ). The flow of nitrogen gas is fixed at $20 \mathrm{ml} \mathrm{second}{ }^{-1}$. 


\section{Powder XRD study}

$\mathrm{XRD}$ analysis was completed on an X-ray diffractometer (Ultima IV, Rigaku, Japan) using K2 $\alpha$ rays with a current of 20 $\mathrm{mA}$ and a voltage of $40 \mathrm{kV}$ in flat plate geometry of 20, ranging from 3 to $70^{\circ}$, and a scan rate of 20 . Pure astaxanthin, PLGA, and astaxanthin-loaded PLGA nanoparticles were tested and analyzed.

\section{FTIR spectroscopy}

The functional group of astaxanthin-loaded PLGA nanoparticles was determined using FTIR. Pure astaxanthin, unprocessed PLGA, and freeze-dried astaxanthin-loaded PLGA nanoparticles were mixed with $\mathrm{KBr}$ at the ratio of $1: 3$, respectively. The mixture was then pressed into a pellet using manual hydraulic press. The disks were then analyzed by FTIR (Varian 640-IR). FTIR spectra were recorded as a mean of 16 scans, in the spectral region ranging from 400 to $4,000 \mathrm{~cm}^{-1}$.

\section{RESULTS AND DISCUSSION}

\section{Particle size and zeta potential analysis}

Nanoparticles can be defined as any particulate that recorded particle size ranging from 1 to $1,000 \mathrm{~nm}$ (Zhao et al., 2014). In order to ascertain the particle size of an optimal formulation, it is crucial to evaluate its size and morphological characteristics. Zeta potential is the surface charge value of a particle of interest. Generally, the more positive or negative the value, the more stable the system. As shown in Table 1 and Figures 1 and 2, the particle size of astaxanthin-loaded PLGA was recorded to be at $142.23 \pm 0.961 \mathrm{~nm}$ and the zeta potential value was $-27.3 \pm 4.67 \mathrm{mV}$. Both values indicated that the astaxanthin nanoparticle particle size was in the nano-range and showed good colloidal stability. These results coincided with a previous study on polymer-based nanoparticles (Mora-Huertas et al., 2010). Due to the high-energy barrier between particles, a zeta value of less than $-10 \mathrm{mV}$ granted good colloidal stability.

\section{SEM}

The morphology analysis was conducted on the newly developed nanoparticles. The SEM photomicrograph in Figure 3 shows that astaxanthin-loaded PLGA nanoparticle size was in between 138.5 and $251.2 \mathrm{~nm}$. Most of the particles were found

Table 1. Analysis by Zetasizer of particle size, zeta potential, and PDI of astaxanthin-loaded PLGA nanoparticle $(n=3)$.

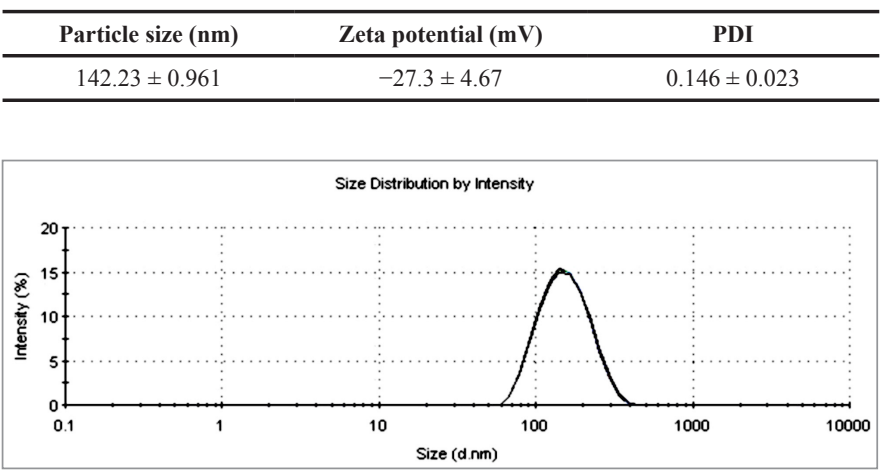

Figure 1. Particle size analysis of astaxanthin-loaded PLGA nanoparticles $(n=3)$. to be spherical in shape with a smooth surface. These results are consistent with the particle size analysis results reported earlier.

\section{Nanoparticle yield and entrapment efficiency}

The yield of nanoparticle and its entrapment efficiency was analyzed to determine the mass of the nanoparticle formed and the efficiency of astaxanthin that is entrapped by PLGA. It was found that the yield achieved for nanoparticle was $18.9 \% \pm$ $1.08 \%$. Despite the low yield reported, the entrapment efficiency showed a high value of $84.375 \% \pm 0.002(n=3)$. Based on the high entrapment efficiency, astaxanthin is suitable to be incorporated into PLGA to form polymeric nanoparticle. This correlates with the study conducted by Mittal et al. (2007), which showed that even at $67 \%$ entrapment efficiency of a drug with almost similar nanoparticle size could result in a significant improvement in drug absorption.

\section{DSC}

DSC is a convenient method to explore the thermal activity of the formulation by providing information on the crystallinity of a particular drug in a system. The analysis was carried out with the curve as a function of temperature instead of time. As shown in Figure 4, pure astaxanthin has the endothermic peak of $227.93^{\circ} \mathrm{C} \pm 0.366^{\circ} \mathrm{C}$, while unprocessed PLGA was at $47.54^{\circ} \mathrm{C} \pm 0.563^{\circ} \mathrm{C}$. However, in the nanoparticle sample, only the

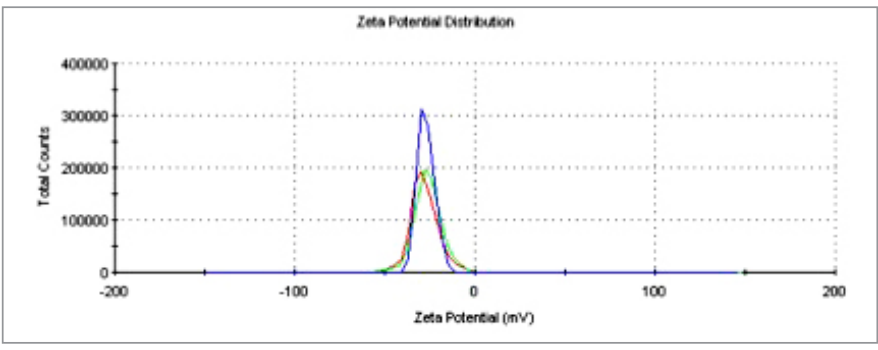

Figure 2. Zeta potential of astaxanthin-loaded PLGA nanoparticles $(n=3)$.

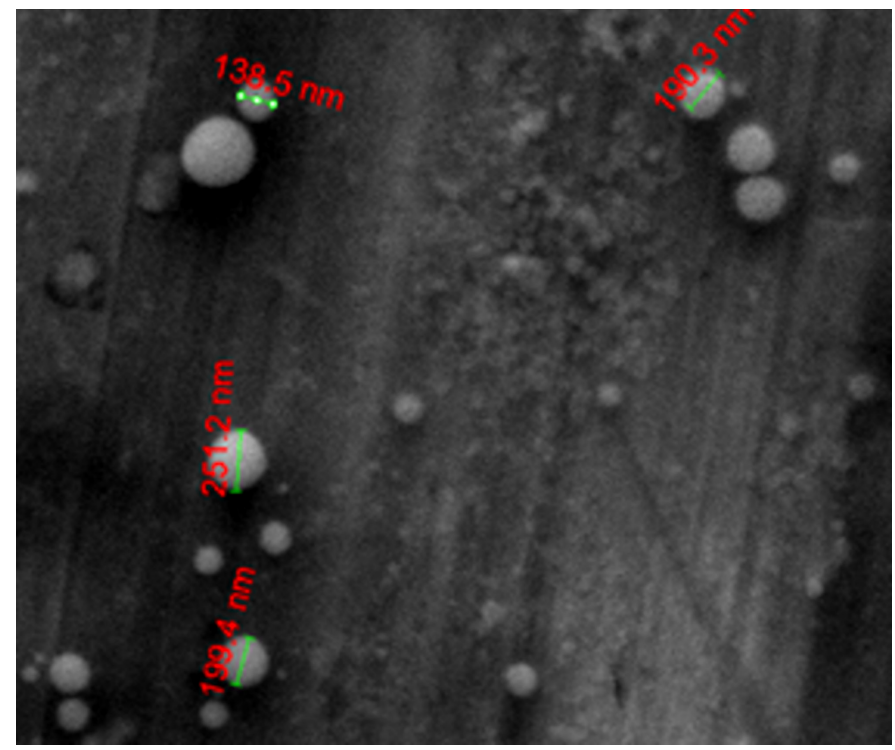

Figure 3. SEM analysis of astaxanthin-loaded PLGA nanoparticles. 


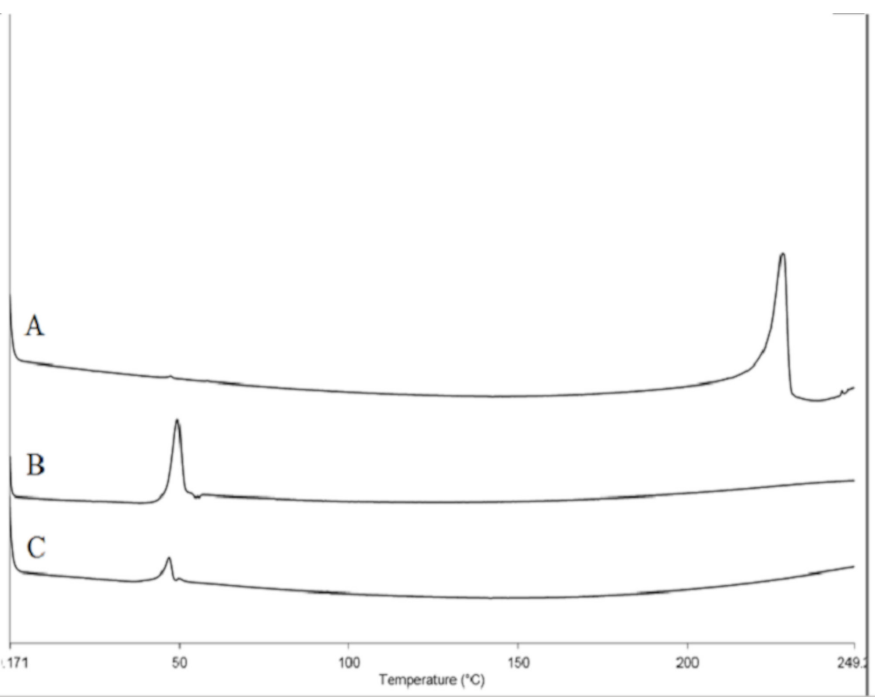

Figure 4. DSC thermogram representing (A) pure astaxanthin, (B) unprocessed PLGA, and (C) astaxanthin-loaded PLGA nanoparticles.

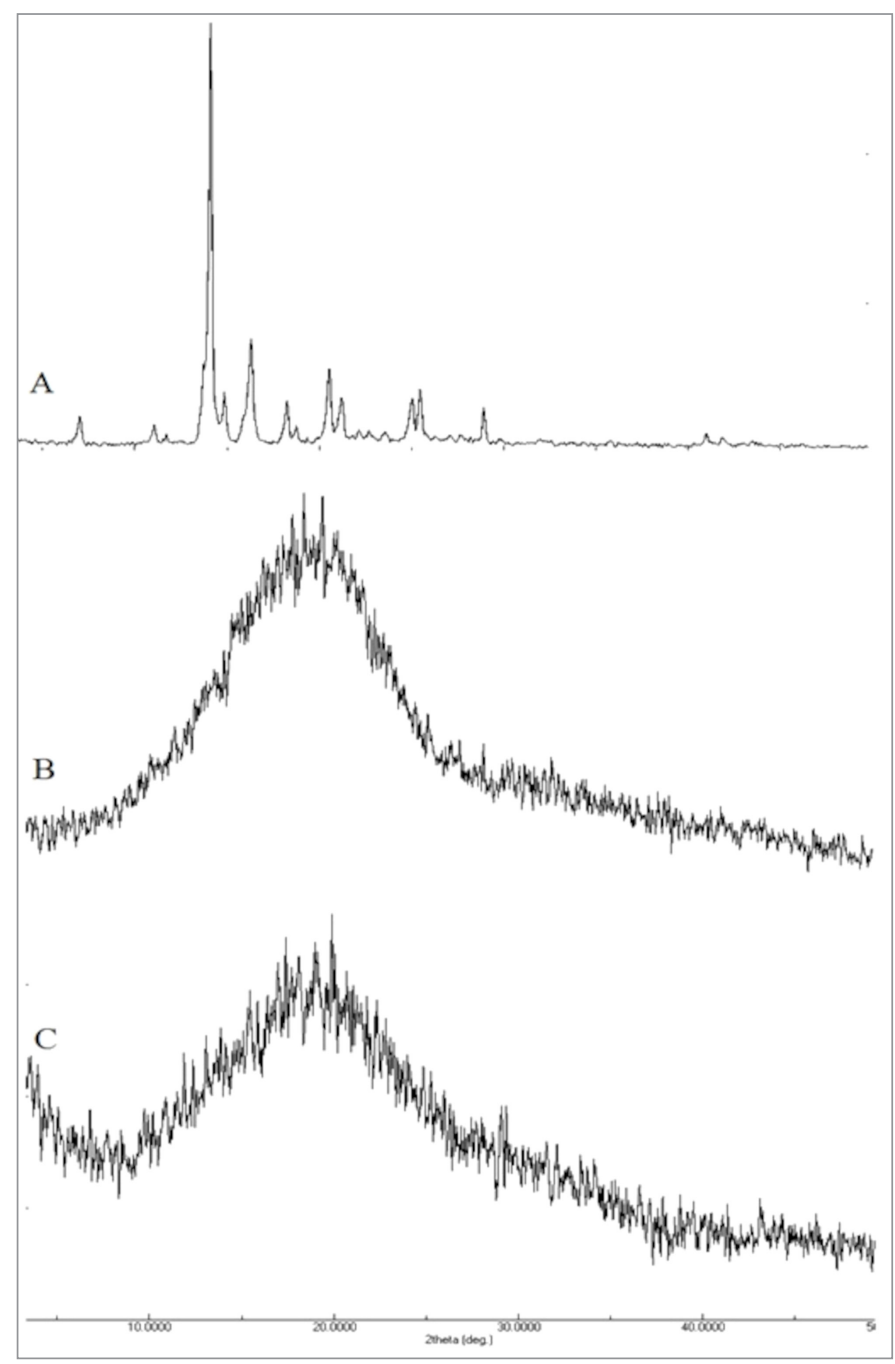

Figure 5. XRD analysis of (A) pure astaxanthin, (B) unprocessed PLGA, and (C) astaxanthin-loaded PLGA nanoparticles.
PLGA peak was present while the astaxanthin peak was absent. The PLGA melting point in the nanoparticle sample was recorded at $46.74 \pm 0.165$, almost a similar temperature as in unprocessed PLGA. The absence of astaxanthin's peak in the nanoparticle curve suggests that the astaxanthin in the system was in the amorphous state or disordered-crystalline phase of a dispersion (Surolia et al., 2012). This phenomenon where the hydrophobic compound or drug entrapped in PLGA becomes amorphous is consistent with the findings reported by other authors (Averineni et al., 2012; Crucho and Barros, 2017).

\section{Powder XRD study}

XRD analysis provides critical information on the crystallinity degree of the formulation, thereby complimenting the DSC analysis results. Figure 5 summarizes the results obtained from the XRD analysis. Astaxanthin showed a highly crystalline structure which is characterized by intense peaks. However, these peaks were diminished in astaxanthin-loaded PLGA nanoparticles. The diminished peaks were due to the fact that the drug has changed from being crystalline to being in an amorphous state in the matrix of the polymer (Sun et al., 2015). This confirmed the results derived from the DSC analysis. In a study carried out by Kim et al. (2008), the amorphous state formed in nanoparticles showed significantly higher solubility compared to unprocessed drug and thereby may improve its bioavailability (Jog and Burgess, 2017).

\section{FTIR spectroscopy}

FTIR analysis made it possible to identify several functional groups present in astaxanthin compound and PLGA

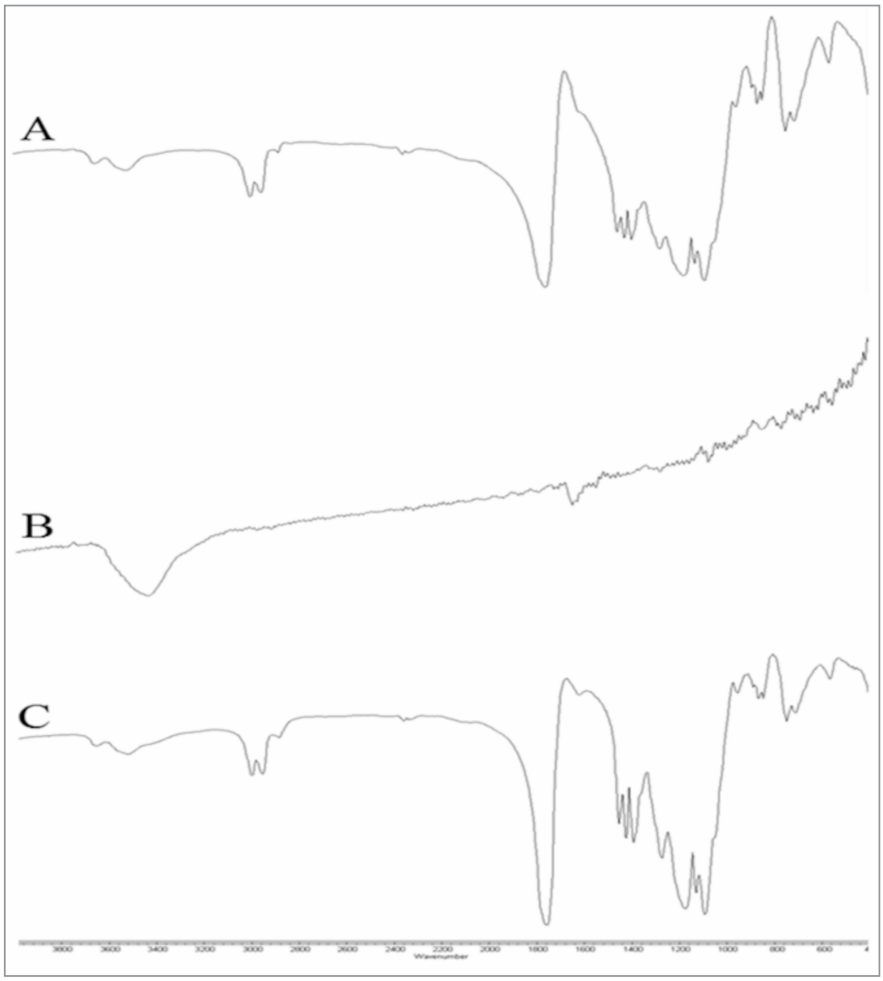

Figure 6. FTIR analysis of (A) unprocessed PLGA, (B) pure astaxanthin, and (C) astaxanthin-loaded PLGA nanoparticle. 
polymer. As shown in Figure 6, there was a broad peak recorded at $3,431.909 \mathrm{~cm}^{-1}$, indicating the presence of the $\mathrm{OH}$ group in the astaxanthin compound. There was also a peak recorded at $1,653.8 \mathrm{~cm}^{-1}$ which indicates the presence of a ketone $(\mathrm{C}=\mathrm{O})$ group. Additionally, PLGA was shown to have a peak at 1,426.611 $\mathrm{cm}^{-1}$ which corresponds to the $\mathrm{C}-\mathrm{H}$ deformation in the $-\mathrm{O}-\mathrm{CH}_{2}-$ structure, which is present only in the glycolic acid structure of PLGA (Kang et al., 2008; Tavakoli et al., 2015). As for the astaxanthin-loaded PLGA nanoparticles, the intensity of peaks that identify the purity of astaxanthin was almost diminished. Because of the environment of the compound, the wavenumber of the ketone group was slightly shifted from its normal range $(1,710$ $\left.1,665 \mathrm{~cm}^{-1}\right)$. This happens when there is another electronegative element attached to the neighbor carbon, and it is most likely that the electron cloud of the element will be affected by the vibration of the interest functional group through Van der Waals repulsion. The same condition was reported by Joshi et al. (2014) and Yuan et al. (2008) where peaks for the drug diminished after the formation of nanoparticle. The phenomenon occurs due to the restriction on the stretching vibrations of bonds in the ketone and $\mathrm{OH}$ group in the polymeric nanoparticle. This finding suggests that optimal incorporation of astaxanthin into PLGA nanoparticle was achieved.

\section{CONCLUSION}

This research focused on the analysis of the physicochemical characteristics of astaxanthin-loaded PLGA nanoparticles. Optimum formulation of astaxanthin-loaded PLGA recorded a particle size of $142.23 \pm 0.961 \mathrm{~nm}$ with a zeta potential value of $-27.3 \pm 4.67 \mathrm{mV}$. The nano-range size of the optimum formulation was further confirmed by SEM photomicrogram. Nanoparticle yield and entrapment efficiency were recorded at $18.9 \% \pm 1.08 \%$ and $84.375 \% \pm 0.002(n=3)$, respectively. DSC thermograph of astaxanthin-loaded nanoparticle was diminished, while XRD study confirmed that the nanoparticles were in an amorphous state. FTIR analysis revealed that the astaxanthin was actually encapsulated inside PLGA, restricting the stretching vibration of the ketone and $\mathrm{OH}$ functional group. Results from these studies suggest that the astaxanthin-loaded PLGA nanoparticles can have the potential to enhance astaxanthin bioavailability.

\section{AUTHOR CONTRIBUTIONS}

All authors made substantial contributions to conception and design, acquisition of data, or analysis and interpretation of data; took part in drafting the article or revising it critically for important intellectual content; agreed to submit to the current journal; gave final approval of the version to be published; and agree to be accountable for all aspects of the work. All the authors are eligible to be an author as per the international committee of medical journal editors (ICMJE) requirements/guidelines.

\section{CONFLICTS OF INTEREST}

The authors report no financial or any other conflicts of interest in this work.

\section{FUNDING}

The authors would like to thank Ministry of Education Malaysia for providing financial assistance (FRGS/1/2012/
SKK02/UITM03/4) and Faculty of Pharmacy UiTM for their support and cooperation in the completion of this study.

\section{ETHICAL APPROVALS}

This study does not involve experiments on animals or human subjects.

\section{PUBLISHER'S NOTE}

This journal remains neutral with regard to jurisdictional claims in published institutional affiliation.

\section{REFERENCES}

Alfarouk KO, Ahmed SBM, Elliott RL, Benoit A, Alqahtani SS, Ibrahim ME, Bashir AHH, Alhoufie STS, Elhassan GO, Wales CC, Schwartz LH, Ali HS, Ahmed A, Forde PF, Devesa J, Cardone RA, Fais S, Harguindey $\mathrm{S}$, Reshkin SJ. The pentose phosphate pathway dynamics in cancer and its dependency on intracellular pH. Metabolites, 2020; 10(7):1-6.

Averineni RK, Shavi GV, Gurram AK, Deshpande PB, Arumugam K, Maliyakkal N, Meka SR, Nayanabhirama U. PLGA 50:50 nanoparticles of paclitaxel: development, in vitro anti-tumor activity in BT549 cells and in vivo evaluation. Bull Mater Sci, 2012; 35(3):319-26.

Crucho CIC, Barros MT. Polymeric nanoparticles: a study on the preparation variables and characterization methods. Mater Sci Eng C, 2017; 80:771-84

Fredenberg S, Wahlgren M, Reslow M, Axelsson A. The mechanisms of drug release in poly(lactic-co-glycolic acid)-based drug delivery systems - a review. Int J Pharm, 2011; 415(1-2):34-52.

Inoue $\mathrm{H}$, Shimamoto $\mathrm{S}$, Takahashi $\mathrm{H}$, Kawashima $\mathrm{Y}$, Wataru S, Ijiri D, Ohtsuka A. Effects of astaxanthin-rich dried cell powder from Paracoccus carotinifaciens on carotenoid composition and lipid peroxidation in skeletal muscle of broiler chickens under thermo-neutral or realistic high temperature conditions. Anim Sci J, 2019; 90(2):229-36.

Ito $\mathrm{N}$, Seki S, Ueda F. The protective role of astaxanthin for UVinduced skin deterioration in healthy people - a randomized, double-blind, placebo-controlled trial. Nutrients, 2018; 10(7):4-6.

Jog R, Burgess DJ. Pharmaceutical amorphous nanoparticles. J Pharm Sci, 2017; 106(1):39-65.

Joshi G, Kumar A, Sawant K. Enhanced bioavailability and intestinal uptake of Gemcitabine $\mathrm{HCl}$ loaded PLGA nanoparticles after oral delivery. Eur J Pharm Sci, 2014; 60:80-9.

Kang Y, Yin G, Ouyang P, Huang Z, Yao Y, Liao X, Chen A, $\mathrm{Pu}$ X. Preparation of PLLA/PLGA microparticles using solution enhanced dispersion by supercritical fluids (SEDS). J Colloid Interface Sci, 2008; 322(1):87-94.

Kavitha K, Kowshik J, Kishore TKK, Baba AB, Nagini S. Astaxanthin inhibits NF- $\mathrm{KB}$ and $\mathrm{Wnt} / \beta$-catenin signaling pathways via inactivation of Erk/MAPK and PI3K/Akt to induce intrinsic apoptosis in a hamster model of oral cancer. Biochim Biophys Acta Gen Subj, 2013; 1830(10):4433-44.

Kim MS, Jin SJ, Kim JS., Park HJ, Song HS, Neubert RHH, Hwang SJ. Preparation, characterization and in vivo evaluation of amorphous atorvastatin calcium nanoparticles using supercritical antisolvent (SAS) process. Eur J Pharm Biopharm, 2008; 69(2):454-65; doi:10.1016/j. ejpb.2008.01.007

$\mathrm{Ku}$ Aizuddin KA, Nurlina MA, Khuriah AH, Foo CS, Meor Mohd Affandi MMR. Development of astaxanthin-loaded biodegradable nanoparticles by nanoprecipitation method. Int J Pharm Technol, 2014; 5(4):5962-72.

Li X, Matsumoto T, Takuwa M, Ali MSES, Hirabashi T, Kondo H, Fujino $\mathrm{H}$. Protective effects of astaxanthin supplementation against ultravioletinduced photoaging in hairless mice. Biomedicines, 2020; 8(2):1-14.

Lobo V, Patil A, Phatak A, Chandra N. Free radicals, antioxidants and functional foods: impact on human health. Pharmacogn Rev, 2010; 4(8):118-26. 
Macarisin D, Droby S, Bauchan G, Wisniewski M. Superoxide anion and hydrogen peroxide in the yeast antagonist-fruit interaction: a new role for reactive oxygen species in postharvest biocontrol? Postharvest Biol Technol, 2010; 58(3):194-202.

Meor Mohd Affandi MMR, Julianto T, Majeed ABA. Development and stability evaluation of astaxanthin nanoemulsion. Asian J Pharm Clin Res, 2011; 4(Suppl 1):143-8.

Meor Mohd Affandi MMR, Abdullah, A, Julianto T, Majeed ABA. Development of simple high performance liquid chromatographic method for the determination of astaxanthin in human plasma. Food Sci Technol Res, 2012;18(1):107-13.

Mittal G, Sahana DK, Bhardwaj V, Ravi Kumar MNV. Estradiol loaded PLGA nanoparticles for oral administration: effect of polymer molecular weight and copolymer composition on release behavior in vitro and in vivo. J Control Release, 2007; 119(1):77-85.

Mora-Huertas CE, Fessi H, Elaissari A. Polymer-based nanocapsules for drug delivery. Int J Pharm, 2010; 385(1-2):113-42.

Raval N, Maheshwari R, Kalyane D, Youngren-Ortiz SR, Chougule MB, Tekade RK. Importance of physicochemical characterization of nanoparticles in pharmaceutical product development. Basic Fundam Drug Deliv, 2018; 369-400; doi:10.1016/B978-0-12-817909-3.00010-8 Sailaja Rao P, Kalva S, Yerramilli A, Mamidi S. Free radicals and tissue damage: role of antioxidants. Free Radic Antioxid, 2011; 1(4):2-7.

Sun S Ben, Liu P, Shao FM, Miao QL. Formulation and evaluation of PLGA nanoparticles loaded capecitabine for prostate cancer. Int J Clin Exp Med, 2015;8(10):19670-81.

Surolia R, Pachauri M, Ghosh PC. Preparation and characterization of monensin loaded PLGA nanoparticles: in vitro antimalarial activity against plasmodium falciparum. J Biomed Nanotechnol, 2012; 8(1):172-81.

Tavakoli E, Mehdikhani-Nahrkhalaji M, Hashemi-Beni B, Zargar-Kharazi A, Kharaziha M. Preparation, characterization and mechanical assessment of poly (lactide-co-glycolide)/hyaluronic acid/ fibrin/bioactive glass nano-composite scaffolds for cartilage tissue engineering applications. Procedia Mat Sci, 2015; 11:124-30.

Tolba SA, Magnuson AD, Sun T, Lei XG. Dietary supplemental microalgal astaxanthin modulates molecular profiles of stress, inflammation, and lipid metabolism in broiler chickens and laying hens under high ambient temperatures. Poult Sci, 2020; 99(10):4853-60.

Tvrdá E, Benko F. Free radicals: what they are and what they do. Pathology, 2020; 3-13; doi:10.1016/B978-0-12-815972-9.00001-9

Yang L, Qiao X, Gu J, Li X, Cao Y, Xu J, Xue C. Influence of molecular structure of astaxanthin esters on their stability and bioavailability. Food Chem, 2020; 343:128497.

Yasui Y, Hosokawa M, Mikami N, Miyashita K, Tanaka T. Dietary astaxanthin inhibits colitis and colitis-associated colon carcinogenesis in mice via modulation of the inflammatory cytokines. Chem Biol Interact, 2011; 193(1):79-87.

Yuan $\mathrm{C}$, Jin Z, Xu X, Zhuang H, Shen W. Preparation and stability of the inclusion complex of astaxanthin with hydroxypropyl- $\beta$ cyclodextrin. Food Chem, 2008; 109(2):264-8.

Zhao L, Seth A, Wibowo N, Zhao CX, Mitter N, Yu C, Middelberg APJ. Nanoparticle vaccines. Vaccine, 2014; 32(3):327-37.

How to cite this article:

Ku Aizuddin KA, Foo CS, Singh GKS, Meor Mohd Affandi

MMR. Physicochemical characterization of astaxanthinloaded PLGA formulation via nanoprecipitation technique. J Appl Pharm Sci, 2021; 11(06):056-061. 